\title{
A tabulation of the bound-state energies of atomic hydrogen
}

\author{
M. Horbatsch and E.A. Hessel, * \\ Department of Physics and Astronomy, York University, Toronto, Ontario M3J 1P3, Canada
}

(Dated: August 31, 2018)

\begin{abstract}
We present tables for the bound-state energies for atomic hydrogen. The tabulated energies include the hyperfine structure, and thus this work extends the work of Rev. Mod. Phys. 84, 1527 (2012), which excludes hyperfine structure. The tabulation includes corrections of the hyperfine structure due to the anomalous moment of the electron, due to the finite mass of the proton, and due to off-diagonal matrix elements of the hyperfine Hamiltonian. These corrections are treated incorrectly in most other works. Simple formulas valid for all quantum numbers are presented for the hyperfine corrections. The tabulated energies have uncertainties of less than $1 \mathrm{kHz}$ for all states. This accuracy is possible because of the recent precision measurement [Nature, 466, 213 (2010); Science, 339, 417] of the proton radius. The effect of this new radius on the energy levels is also tabulated, and the energies are compared to precision measurements of atomic hydrogen energy intervals.
\end{abstract}

PACS numbers: \pacs\{31.15.ac,31.15.1j, 06.20.Jr,12.20.-m, 14.20.Dh\}

\section{INTRODUCTION}

The current state of the theoretical knowledge of the bound-state energy levels of atomic hydrogen has been presented in the CODATA analysis of Ref. [1]. Here we expand on that work, by also including the hyperfine structure. Because of a recent precise determination [2, 3] of the rms proton charge radius $R_{\mathrm{p}}$, obtained from measurements of the $n=2$ intervals in muonic hydrogen, all of the atomic hydrogen energy levels can now be determined to an accuracy of better than $1 \mathrm{kHz}$. In this work, we present tables for the energies $E_{n \ell j f}$ of $\left|n l j f m_{f}\right\rangle$ bound states of atomic hydrogen. These tables also indicate how the energy of each state is affected by the new value of $R_{\mathrm{p}}$ and by the new determination of the Rydberg constant that results from this new $R_{\mathrm{p}}$ value.

The present work was performed because of the need for precision energy levels for the analysis of an ongoing measurement of the hydrogen $n=2$ Lamb shift, for which the hyperfine structure and fine structure must be carefully considered. The tabulation is mostly based on the theoretical and experimental work of others, and is intended as a practical resource.

\section{OVERVIEW}

To date, only three intervals in atomic hydrogen have been measured to an accuracy of better than $1 \mathrm{kHz}$ :

$\nu\left(1 \mathrm{~S}_{1 / 2} f=1 \rightarrow 2 \mathrm{~S}_{1 / 2} f=1\right): 2466061102474.806(10) \mathrm{kHz}$, $\nu\left(1 \mathrm{~S}_{1 / 2} f=0 \rightarrow 1 \mathrm{~S}_{1 / 2} f=1\right): 1420405.751768(1) \mathrm{kHz}$, $\nu\left(2 \mathrm{~S}_{1 / 2} f=0 \rightarrow 2 \mathrm{~S}_{1 / 2} f=1\right): 177556.8343(67) \mathrm{kHz}$,

where the first and the last values were measured [4, 5] by the Hänsch group, and the $1 \mathrm{~S}_{1 / 2}$ hyperfine interval is based on an analysis by Karshenboim 6] of all measurements of this interval. Theoretical predictions are needed to determine all other intervals to an accuracy of $<1 \mathrm{kHz}$. In addition, precise values of fundamental constants are required. The most important constant needed is the Rydberg constant $\left(R_{\infty}\right)$. As will be discussed in Section $\mathrm{V}$ this constant can be obtained from a combination of the theoretical predictions for the hydrogen atom and the measurements of Eq. (1).

The second most important constant is the finestructure constant $(\alpha)$, the value of which is known from a comparison between theory and measurements of the magnetic moment of the electron. For this work, we use the CODATA 2014 value of

$$
\alpha=1 / 137.035999139(31),
$$

which is derived almost entirely from the electron magnetic moment. The proton's mass, magnetic moment and rms charge ratio are also needed. The CODATA 2014 values for these quantities are

$$
\begin{aligned}
m_{\mathrm{p}} / m_{\mathrm{e}} & =1836.15267389(17), \\
g_{\mathrm{p}} & =5.585694702(17), \\
\text { and } R_{\mathrm{p}} & =0.8751(61) \mathrm{fm} .
\end{aligned}
$$

A more precise determination of the rms charge radius has been obtained using measurements [2, 3] of the $n=2$ levels of muonic hydrogen by the CREMA collaboration:

$$
R_{\mathrm{p}}=0.84087(39) \mathrm{fm} .
$$

This value differs significantly from the CODATA 2014 determination. We use this more precise determination of $R_{\mathrm{p}}$ in this work, but also tabulate the shifts in the bound-state hydrogen energies that would result if the CODATA 2014 value were used.

The other two required constants are the Compton wavelength, $\lambda_{C}$, and the muon mass, $m_{\mu}$, which have CODATA 2014 values of

$$
\begin{aligned}
\lambda_{C}=\frac{\lambda_{C}}{2 \pi} & =386.15926764(18) \mathrm{fm}, \\
\text { and } m_{\mu} / m_{\mathrm{e}} & =206.7682826(46) .
\end{aligned}
$$


The constants of Eqs. (2), (3), (4), (6), (7), and (8) are all determined using physical systems other than atomic hydrogen, and therefore should be considered to be external inputs to the theory. Eqs. (2), (3), (4) (7), and (8) are known with sufficient precision as to lead to uncertainties in the hydrogen energies of much less than $0.1 \mathrm{kHz}$. The proton charge radius $R_{\mathrm{p}}$ of Eq. (6), however, despite its more precise determination, is still one of the leading causes for uncertainty in the hydrogen energies.

The binding energy of an $\left|n \ell j f m_{f}\right\rangle$ state of hydrogen can be written as

$$
E_{n \ell j f}=E_{n}^{(\mathrm{g})}+E_{n \ell j}^{(\mathrm{fs})}+E_{n \ell j f}^{(\mathrm{hfs})}
$$

where

$$
E_{n}^{(\mathrm{g})}=-\frac{\mathcal{R}}{n^{2}} \frac{m_{\mathrm{r}}}{m_{\mathrm{e}}}
$$

is the gross structure, $E_{n \ell j}^{(\mathrm{fs})}$ is the fine structure contribution, and $E_{n \ell j f}^{(\mathrm{hfs})}$ is the hyperfine structure contribution. The energies are, of course, independent of $m_{f}$ in the absence of an external field. Here, $\mathcal{R}=c R_{\infty}, c$ is the speed of light, $R_{\infty}$ is the Rydberg constant, $m_{\mathrm{r}}=m_{\mathrm{e}} m_{\mathrm{p}} / M$ is the reduced mass, $M=m_{\mathrm{e}}+m_{\mathrm{p}}$, and $m_{\mathrm{e}}$ and $m_{\mathrm{p}}$ are the electron and proton masses, respectively, For this work, Planck's constant $h$ is suppressed, and all energies are given in frequency units.

\section{FINE-STRUCTURE ENERGY CONTRIBUTIONS}

The values of $E_{n \ell j}^{(\mathrm{fs})}$ are discussed in detail in Ref. [1]:

$$
\begin{aligned}
E_{n \ell j}^{(\mathrm{fs})} & =\Delta E_{M}+E_{\mathrm{S}}+E_{\mathrm{R}}+E_{\mathrm{SE}}^{(2)}+E_{\mathrm{VP}}^{(2)} \\
& +E^{(4)}+E^{(6)}+E_{\mathrm{RR}}+E_{\mathrm{SEN}}+E_{\mathrm{NS}},
\end{aligned}
$$

where $\Delta E_{M}$ gives the mass-corrected Dirac fine structure, $E_{\mathrm{S}}$ and $E_{\mathrm{R}}$ are relativistic recoil corrections, $E_{\mathrm{SE}}^{(2)}$ and $E_{\mathrm{VP}}^{(2)}$ are self-energy and vacuum polarization quantum-electrodynamics (QED) corrections, $E^{(4)}$ and $E^{(6)}$ are higher-order QED corrections, $E_{\mathrm{RR}}$ and $E_{\mathrm{SEN}}$ are small QED corrections due to the finite mass of the proton, and $E_{\mathrm{NS}}$ is the correction for the size (rms charge radius) of proton. For completeness, we include the formulas given in Ref. [1] that give contributions which are significant to this work, leaving out energy terms and corrections that lead to contributions of $<0.025 \mathrm{kHz}$ to our tabulated values. Because of the precisely-measured 1S-2S interval of Eq. (11), $E^{(\mathrm{fs})}$ for the 1S state energy needs to be determined less accurately, and is only necessary for the determination of the Rydberg constant, as will be discussed in Section $\mathrm{V}$.

From Ref. [1], we obtain:

$$
\begin{aligned}
\Delta E_{M}= & E_{M}-M c^{2}-E_{n}^{(\mathrm{g})}=\frac{2\left[f_{n j}-1\right]-\left[f_{n j}-1\right]^{2} \frac{m_{\mathrm{r}}}{M}}{\alpha^{2}} \frac{\mathcal{R} m_{\mathrm{r}}}{m_{\mathrm{e}}} \\
& +\frac{\mathcal{R}}{n^{2}} \frac{m_{\mathrm{r}}}{m_{\mathrm{e}}}+\frac{1-\delta_{\ell 0}}{2 \ell+1} \frac{\alpha^{2} \mathcal{R} m_{\mathrm{e}}^{2}}{\kappa_{\ell j} n^{3} m_{\mathrm{p}}^{2}},
\end{aligned}
$$

$$
\begin{gathered}
E_{\mathrm{S}}=\frac{2 m_{\mathrm{r}}^{3} \alpha^{3} \mathcal{R}}{m_{\mathrm{e}}^{2} m_{\mathrm{p}} \pi n^{3}}\left\{\frac{\delta_{\ell 0}}{3} \lambda-\frac{8}{3} \beta_{n \ell}-\frac{\delta_{\ell 0}}{9}-\frac{7}{3} a_{n \ell}-2 \delta_{\ell 0} \frac{m_{\mathrm{e}}}{m_{\mathrm{p}}}\right\} \\
E_{\mathrm{R}}=\frac{2 m_{\mathrm{e}} \alpha^{4} \mathcal{R}}{m_{\mathrm{p}} \pi n^{3}}\left\{D_{60}^{(n \ell)}-\frac{11 \delta_{\ell 0}}{60} \alpha \lambda^{2}+D_{71}^{(n \ell j)} \alpha \lambda\right\}, \\
E_{\mathrm{SE}}^{(2)}=\frac{2 m_{\mathrm{r}}^{3} \alpha^{3} \mathcal{R}}{m_{\mathrm{e}}^{3} \pi n^{3}}\left\{\frac{4 \delta_{\ell 0}}{3} \lambda-\frac{4 \beta_{n \ell}}{3}+\frac{10 \delta_{\ell 0}}{9}-\frac{\left(1-\delta_{\ell 0}\right)}{2 \kappa_{\ell j}(2 \ell+1)} \frac{m_{\mathrm{e}}}{m_{\mathrm{r}}}\right. \\
+\left(\frac{139}{32}-2 \ln 2\right) \pi \delta_{\ell 0} \alpha-\delta_{\ell 0} \alpha^{2} \lambda^{2} \\
\left.+A_{61}^{(n \ell j)} \alpha^{2} \lambda+G_{\mathrm{SE}}^{(n \ell j)} \alpha^{2}\right\},
\end{gathered}
$$

$$
\begin{aligned}
& E_{\mathrm{VP}}^{(2)}=\frac{2 m_{\mathrm{r}}^{3} \alpha^{3} \mathcal{R}}{m_{\mathrm{e}}^{3} \pi n^{3}}\left\{\left[-\frac{4}{15}\left(1+1.67 \frac{m_{\mathrm{e}}^{2}}{m_{\mu}^{2}}\right)+\frac{5 \pi}{48} \alpha-\frac{2}{15} \alpha^{2} \lambda\right.\right. \\
&\left.\left.+\left(\frac{19}{45}-\frac{\pi^{2}}{27}\right) \alpha^{2}\right] \delta_{\ell 0}+G_{\mathrm{VP}}^{(1)(n \ell j)} \alpha^{2}\right\}, \\
& E^{(4)}=\frac{2 m_{\mathrm{r}}^{3} \alpha^{4} \mathcal{R}}{m_{\mathrm{e}}^{3} \pi^{2} n^{3}}\left\{0.53894 \delta_{\ell 0}+\frac{0.3285\left(1-\delta_{\ell 0}\right)}{\kappa_{\ell j}(2 \ell+1)} \frac{m_{\mathrm{e}}}{m_{\mathrm{r}}}\right. \\
&- 21.554 \delta_{\ell 0} \alpha-\frac{8 \delta_{\ell 0}}{27} \alpha^{2} \lambda^{3}+B_{62}^{(n \ell)} \alpha^{2} \lambda^{2} \\
&+\left.B_{61}^{(n \ell j)} \alpha^{2} \lambda+B_{60}^{(n \ell j)} \alpha^{2}\right\},
\end{aligned}
$$

$$
\begin{gathered}
E^{(6)}=\frac{2 m_{\mathrm{r}}^{3} \alpha^{5} \mathcal{R}}{m_{\mathrm{e}}^{3} \pi^{3} n^{3}}\left\{0.418 \delta_{\ell 0}-\frac{1.2\left(1-\delta_{\ell 0}\right)}{\kappa_{\ell j}(2 \ell+1)} \frac{m_{\mathrm{e}}}{m_{\mathrm{r}}}+C_{50} \alpha\right\}, \\
E_{\mathrm{NS}}=\frac{4 m_{\mathrm{r}}^{3} \alpha^{2} R_{\mathrm{p}}^{2} \mathcal{R}}{3 m_{\mathrm{e}}^{3} \lambda_{C}^{2} n^{3}}\left\{1-\alpha^{2} \ln \frac{\alpha R_{\mathrm{p}}}{n \lambda_{C}}-1.8 \alpha^{2}\right\} \delta_{\ell 0}, \\
E_{\mathrm{RR}}=\frac{2 m_{\mathrm{r}}^{3} \alpha^{4} \mathcal{R}}{m_{\mathrm{p}} m_{\mathrm{e}}^{2} \pi^{2} n^{3}} \delta_{\ell 0}\left\{-13.47+\frac{2}{3} \pi \alpha \lambda^{2}+\Delta^{\mathrm{RR}} \alpha \lambda\right\},
\end{gathered}
$$

and

$$
E_{\mathrm{SEN}}=\frac{8 m_{\mathrm{r}}^{3} \alpha^{3} \mathcal{R}}{3 m_{\mathrm{p}}^{2} m_{\mathrm{e}} \pi n^{3}}\left\{\delta_{\ell 0} \ln \frac{m_{\mathrm{p}}}{m_{\mathrm{e}} \alpha^{2}}-\beta_{n \ell}\right\} .
$$

Here, $\lambda=\ln \left(\alpha^{-2} m_{\mathrm{e}} / m_{\mathrm{r}}\right), \quad \delta_{\ell 0}$ is the Kronecker delta, $\kappa_{\ell j}=(\ell-j)(2 j+1), \quad f_{n j}=\left[1+\alpha^{2}(n-\delta)^{-2}\right]^{-1 / 2} \quad$ in Eq. (12) (with $\left.\delta=j+\frac{1}{2}-\left[\left(j+\frac{1}{2}\right)^{2}-\alpha^{2}\right]^{1 / 2}\right), \beta_{n \ell}$ are the Bethe logarithms (tabulated in Ref. [7]),

$$
\begin{gathered}
a_{n \ell}=-2\left[\ln \frac{2}{n}+\sum_{i=1}^{n} \frac{1}{i}+1-\frac{1}{2 n}\right] \delta_{\ell 0}+\frac{1-\delta_{\ell 0}}{\ell(\ell+1)(2 \ell+1)},(22) \\
D_{60}^{(n \ell)}=\left(4 \ln 2-\frac{7}{2}\right) \pi \delta_{\ell 0}+\frac{\left[3-\frac{\ell(\ell+1)}{n^{2}}\right] 2 \pi\left(1-\delta_{\ell 0}\right)}{\left(4 \ell^{2}-1\right)(2 \ell+3)}, \\
A_{61}^{(n \ell j)}=\left[\left(\sum_{i=1}^{n} \frac{4}{i}\right)+\frac{28}{3} \ln 2-4 \ln n-\frac{601}{180}-\frac{77}{45 n^{2}}\right] \delta_{\ell 0} \\
+\left(1-\frac{1}{n^{2}}\right)\left(\frac{2}{15}+\frac{\delta_{j \frac{1}{2}}}{3}\right) \delta_{\ell 1}+\frac{8\left(3 n^{2}-\ell(\ell+1)\right)\left(1-\delta_{\ell 0}\right)}{3 n^{2} \ell\left(4 \ell^{2}-1\right)(\ell+1)(2 \ell+3)},
\end{gathered}
$$


and

$$
B_{62}^{(n \ell)}=\frac{16}{9}\left[1.067+\psi(n)-\ln n-\frac{1}{n}+\frac{1}{4 n^{2}}\right] \delta_{\ell 0}+\frac{4 \delta_{\ell 1}}{27} \frac{n^{2}-1}{n^{2}},
$$

with $\psi$ being the digamma function. The values of $G_{\mathrm{SE}}^{(n \ell j)}$ of Eq. (15), $G_{\mathrm{VP}}^{(1)(n \ell j)}$ of Eq. (16), and $B_{61}^{(n \ell j)}$ and $B_{60}^{(n \ell j)}$ of Eq. (17) are discussed in Ref. 1], and the tabulated values (along with values given in Refs. [8-12] and simple extrapolations and interpolations) are sufficiently precise for the current work. The values of $D_{71}^{(n \ell j)}$ are needed only for the lowest-lying states, and have recently been calculated in Ref. [13].

Although many uncertainties to the fine-structure energy contributions $E_{n \ell j}^{(\mathrm{fs})}$ are detailed in Ref. [1], only four uncertainties dominate and need to be considered in this work. The first comes from an uncertainty of \pm 19.7 in the $\ell=0$ coefficients $B_{60}^{(n \ell j)}$ of Eq. (17), which leads to an uncertainty of $\delta_{\ell 0}(2.0 \mathrm{kHz}) / n^{3}$. The second comes from the uncertainty in $R_{\mathrm{p}}$ (of Eq. (6)) in Eq. (19), which contributes $\delta_{\ell 0}(1.0 \mathrm{kHz}) / n^{3}$. The third comes from the coefficient $C_{50}$ of Eq. (18), which is presumed to be $\pm 30 \delta_{\ell 0}$, and which leads to an uncertainty of $\delta_{\ell 0}(1.0 \mathrm{kHz}) / n^{3}$. The fourth comes from the $\Delta^{\mathrm{RR}}$ coefficient of Eq. (20), which is presumed to be \pm 10 , thus leading to an uncertainty of $\delta_{\ell 0}(0.7 \mathrm{kHz}) / n^{3}$. An additional uncertainty in Eq. (14), has now been resolved by Ref. [13] and does not need to be included. These four dominant uncertainties all have the same dependence on $n$ and $\ell$ and therefore can be added in quadrature to give a combined uncertainty of $\delta_{\ell 0}(2.6 \mathrm{kHz}) / n^{3}$. All other uncertainties listed in Ref. [1] are more than an order of magnitude smaller. The values of $E_{n \ell j}^{(\mathrm{fs})}$ for the lowest-lying states, along with their uncertainties are listed in Table —

\section{HYPERFINE STRURCTURE} by

The hyperfine contributions to the energies are given

$$
E_{n \ell j f}^{(\mathrm{hfs})}=\delta_{\ell 0}\left(f-\frac{3}{4}\right) \frac{\Delta E_{1 S}^{\mathrm{hfs}}+\Delta_{n}}{n^{3}}+\left(1-\delta_{\ell 0}\right)\left(E_{\mathrm{diag}}^{\mathrm{hfs}}+\Delta E^{\mathrm{hfs}}\right) .
$$

For $\ell=0$ states, where the structure of the nucleus affects the hyperfine structure, $E_{n \ell j f}^{(\mathrm{hfs})}$ is determined using the precise measurement of the ground-state hyperfine interval $\Delta E_{1 S}^{\mathrm{hfs}}$ of Eq. (1). For $n=2, \Delta_{2}=48.922(27) \mathrm{kHz}$ can be determined directly from the measured interval of Eq. (1). For higher $n$, the correction $\Delta_{n}$ is discussed in detail in Ref. [14], and, to the accuracy required here, is given by

$$
\Delta_{n}=\frac{8}{3} g_{\mathrm{p}} \alpha^{4} \mathcal{R} \frac{m_{\mathrm{e}}}{m_{\mathrm{p}}}\left(\frac{1}{3}+\frac{3}{2 n}-\frac{11}{6 n^{2}}\right)
$$

TABLE I. Fine-structure energies $E_{n \ell j}^{(\mathrm{fs})}$ for the lowest-lying states of atomic hydrogen. Uncertainties in the last digits are shown in parentheses. These values were determined using the Rydberg constant obtained in Section $\nabla$ however, using the CODATA 2014 value instead would not change the values, since the resulting changes would be at the level of $1 \mathrm{~Hz}$ or less.

\begin{tabular}{lccc}
\hline \hline & $E_{n \ell j}^{(\mathrm{fs})}(\mathrm{kHz})$ & & $E_{n \ell j}^{(\mathrm{fs})}(\mathrm{kHz})$ \\
\hline $1 \mathrm{~S}_{1 / 2}$ & $-35625530.5(2.6)$ & & \\
$2 \mathrm{~S}_{1 / 2}$ & $-12636029.4(3)$ & & \\
$2 \mathrm{P}_{1 / 2}$ & $-13693861.6(0)$ & $2 \mathrm{P}_{3 / 2}$ & $-2724820.1(0)$ \\
$3 \mathrm{~S}_{1 / 2}$ & $-4552716.0(1)$ & & \\
$3 \mathrm{P}_{1 / 2}$ & $-4867590.3(0)$ & $3 \mathrm{P}_{3 / 2}$ & $-1617501.0(0)$ \\
$3 \mathrm{D}_{3 / 2}$ & $-1622832.7(0)$ & $3 \mathrm{D}_{5 / 2}$ & $-539495.5(0)$ \\
$4 \mathrm{~S}_{1 / 2}$ & $-2091332.8(0)$ & & \\
$4 \mathrm{P}_{1 / 2}$ & $-2224408.7(0)$ & $4 \mathrm{P}_{3 / 2}$ & $-853278.9(0)$ \\
$4 \mathrm{D}_{3 / 2}$ & $-855566.5(0)$ & $4 \mathrm{D}_{5 / 2}$ & $-398533.1(0)$ \\
$4 \mathrm{~F}_{5 / 2}$ & $-399342.4(0)$ & $4 \mathrm{~F}_{7 / 2}$ & $-170827.1(0)$ \\
$5 \mathrm{~S}_{1 / 2}$ & $-1123202.3(0)$ & & \\
$5 \mathrm{P}_{1 / 2}$ & $-1191397.0(0)$ & $5 \mathrm{P}_{3 / 2}$ & $-489379.3(0)$ \\
$5 \mathrm{D}_{3 / 2}$ & $-490561.2(0)$ & $5 \mathrm{D}_{5 / 2}$ & $-256560.1(0)$ \\
$5 \mathrm{~F}_{5 / 2}$ & $-256977.8(0)$ & $5 \mathrm{~F}_{7 / 2}$ & $-139977.9(0)$ \\
$5 \mathrm{G}_{7 / 2}$ & $-140196.4(0)$ & $5 \mathrm{G}_{9 / 2}$ & $-69996.6(0)$ \\
$6 \mathrm{~S}_{1 / 2}$ & $-670236.8(0)$ & & \\
$6 \mathrm{P}_{1 / 2}$ & $-709720.8(0)$ & $6 \mathrm{P}_{3 / 2}$ & $-303461.0(0)$ \\
$6 \mathrm{D}_{3 / 2}$ & $-304148.6(0)$ & $6 \mathrm{D}_{5 / 2}$ & $-168731.3(0)$ \\
$6 \mathrm{~F}_{5 / 2}$ & $-168974.3(0)$ & $6 \mathrm{~F}_{7 / 2}$ & $-101266.0(0)$ \\
$6 \mathrm{G}_{7 / 2}$ & $-101393.0(0)$ & $6 \mathrm{G}_{9 / 2}$ & $-60768.1(0)$ \\
$6 \mathrm{H}_{9 / 2}$ & $-60846.8(0)$ & $6 \mathrm{H}_{11 / 2}$ & $-33763.6(0)$ \\
\hline \hline
\end{tabular}

For $\ell \neq 0$, nuclear effects are not significant, and the dominant diagonal term $E_{\text {diag }}^{\mathrm{hfs}}$ is given by

$$
\begin{aligned}
E_{\mathrm{diag}}^{\mathrm{hfs}}= & g_{\mathrm{p}} \frac{\alpha^{2} \mathcal{R}}{n^{3}} \frac{m_{\mathrm{r}}^{3}}{m_{\mathrm{e}}^{3}} \frac{m_{\mathrm{e}}}{m_{\mathrm{p}}} \frac{f(f+1)-j(j+1)-\frac{3}{4}}{(2 \ell+1) j(j+1)}\left\{1+\frac{a_{\mathrm{e}}}{2 \kappa_{\ell j}}\right. \\
+ & \frac{g_{\mathrm{p}}-1}{g_{\mathrm{p}}} \frac{m_{\mathrm{e}}}{m_{\mathrm{p}}} \frac{2 \kappa_{\ell j}-1}{2 \kappa_{\ell j}}+\alpha^{2}\left[\frac{3(2 j+1)^{2}-1}{2(2 j+1)^{2} j(j+1)}\right. \\
& \left.\left.+\frac{3}{n(2 j+1)}+\frac{3-8 \kappa_{\ell j}}{2 n^{2}\left(2 \kappa_{\ell j}-1\right)}\right]\right\}
\end{aligned}
$$

The $a_{e}=\left(g_{e}-2\right) / 2$ electron anomalous moment corrections do not apply to the $\vec{I} \cdot \vec{L}$ term in the hyperfine Hamiltonian, and this leads to the $2 \kappa_{\ell j}$ denominator of the $a_{e}$ term. The $a_{e}$ correction is included (for the $2 \mathrm{P}$ states) in Ref. [15], is included to first order in $\alpha$ in Ref. [16], but is included incorrectly (without the $2 \kappa_{\ell j}$ in the denominator) in Ref. [17] and Ref. [18]. Given the size of the hyperfine structure, it is sufficient for this work to approximate $a_{e}$ by its lowest-order term: $\alpha /(2 \pi)$.

The correction proportional to $\left(\left(g_{\mathrm{p}}-1\right) / g_{\mathrm{p}}\right)\left(m_{\mathrm{e}} / m_{\mathrm{p}}\right)$ results from the interaction of the proton's orbital motion with its spin. This term is included (for the $2 \mathrm{P}$ states) in Ref. [15], but is given incorrectly for the $2 \mathrm{P}$ state in Eqs. (27) and (28) of Ref. [19] for muonic hydrogen, where the term is even more important. The term is correctly included for muonic hydrogen in Table II of Ref. [19] and in Ref. [20]. The term is omitted 
TABLE II. Hyperfine-structure energies $E_{n \ell j f}^{(\mathrm{hfs})}$ for the lowestlying states of atomic hydrogen. All values are uncertain by less than $0.1 \mathrm{kHz}$. The precisely measured values are given for the $1 \mathrm{~S}$ and $2 \mathrm{~S}$ states.

\begin{tabular}{lcccc}
\hline \hline & $f$ & $E_{n \ell j f}^{(\mathrm{hfs})}(\mathrm{kHz})$ & $f$ & $E_{n \ell j f}^{(\mathrm{hfs})}(\mathrm{kHz})$ \\
\hline $1 \mathrm{~S}_{1 / 2}$ & 0 & $-1065304.3138260(8)$ & 1 & $355101.4379420(3)$ \\
$2 \mathrm{~S}_{1 / 2}$ & 0 & $-133167.6257(51)$ & 1 & $44389.2086(17)$ \\
$2 \mathrm{P}_{1 / 2}$ & 0 & -44379.0 & 1 & 14790.5 \\
$2 \mathrm{P}_{3 / 2}$ & 1 & -14781.3 & 2 & 8870.3 \\
$3 \mathrm{~S}_{1 / 2}$ & 0 & -39457.0 & 1 & 13152.3 \\
$3 \mathrm{P}_{1 / 2}$ & 0 & -13149.4 & 1 & 4382.4 \\
$3 \mathrm{P}_{3 / 2}$ & 1 & -4379.7 & 2 & 2628.2 \\
$3 \mathrm{D}_{3 / 2}$ & 1 & -2629.2 & 2 & 1577.4 \\
$3 \mathrm{D}_{5 / 2}$ & 2 & -1576.9 & 3 & 1126.4 \\
$4 \mathrm{~S}_{1 / 2}$ & 0 & -16645.9 & 1 & 5548.6 \\
$4 \mathrm{P}_{1 / 2}$ & 0 & -5547.4 & 1 & 1848.8 \\
$4 \mathrm{P}_{3 / 2}$ & 1 & -1847.7 & 2 & 1108.8 \\
$4 \mathrm{D}_{3 / 2}$ & 1 & -1109.2 & 2 & 665.5 \\
$4 \mathrm{D}_{5 / 2}$ & 2 & -665.3 & 3 & 475.2 \\
$4 \mathrm{~F}_{5 / 2}$ & 2 & -475.3 & 3 & 339.5 \\
$4 \mathrm{~F}_{7 / 2}$ & 3 & -339.4 & 4 & 264.0 \\
\hline \hline
\end{tabular}

in Ref. [16], Ref. [21], Ref [18] and Ref. [17]. This masscorrection term contributes $13 \mathrm{kHz}$ to the $2 \mathrm{P}_{1 / 2}$ hyperfine structure, and thus must certainly be included at the accuracy of this work. The general form for this correction (as a function of $n, \ell$, and $j$ ) does not seem to appear previously in the literature.

The correction proportional to $\alpha^{2}$ in Eq. (28) is a relativistic correction which is given in Ref. [16]. Higherorder corrections are also considered in that work, but are insignificant at the level of this work.

The smaller $\Delta E^{\mathrm{hfs}}$ contribution comes from an off-diagonal element of the hyperfine Hamiltonian. This element causes a very slight mixing between the $\left|n \ell j=\ell-\frac{1}{2} f=\ell m_{f}\right\rangle$ state and the $\left|n \ell j=\ell+\frac{1}{2} f=\ell m_{f}\right\rangle$ state, and its contribution to the energy can be determined to sufficient accuracy by the expression from second-order perturbation theory:

$$
\begin{aligned}
\Delta E^{\mathrm{hfs}} & =\frac{\left|\left\langle n \ell j f=\ell m_{f}\left|H_{\mathrm{hfs}}\right| n \ell j^{\prime} f=\ell m_{f}\right\rangle\right|^{2}}{E_{n \ell j}^{\mathrm{fs})}-E_{n \ell j^{\prime}}^{(\mathrm{fs})}} \\
& =\frac{2 \alpha^{2} \mathcal{R}}{n^{3}} \frac{m_{\mathrm{e}}^{2}}{m_{\mathrm{p}}^{2}} g_{\mathrm{p}}^{2} \frac{(j-\ell) \delta_{f \ell}}{(2 \ell+1)^{4}} .
\end{aligned}
$$

This expression for $\Delta E^{\mathrm{hfs}}$ was first given (for the $2 \mathrm{P}$ states) in Ref. [15]. Its general form does not appear to be presented in the literature, and an incorrect form (with an incorrect dependence on $\ell$ ) is used in the tabulation of Ref. [17]. The contribution used in Ref. [17] for the off-diagonal contributions is too large by a factor of $\ell^{2}(\ell+1)^{2}(2 \ell+1)^{2} / 36$ (a factor of $25,196,900$, and 3025 for D, F, G, and $\mathrm{H}$ states, respectively).

The values of $E_{n \ell j f}^{(\mathrm{hfs})}$ of Eq. (26) are listed in Table II The tabulated values include the contributions Eq. (29). In all cases the values can be determined to better than $0.1 \mathrm{kHz}$.

\section{GROSS STRUCTURE}

The gross structure $E^{(\mathrm{g})}$ of Eq. (10) requires a precise value for the Rydberg constant. This value can be obtained by equating the precise measured value of Eq. (1) for the $1 \mathrm{~S}_{1 / 2} f=1 \rightarrow 2 \mathrm{~S}_{1 / 2} f=1$ interval to Eq. (9), with the values of $E_{n \ell j}^{(\mathrm{fs})}$ and $E_{n \ell j f}^{(\mathrm{hfs})}$ obtained in the previous sections:

$$
\begin{aligned}
& 2466061102474.806(10) \mathrm{kHz}=\frac{3}{4} \mathcal{R} \frac{m_{\mathrm{r}}}{m_{\mathrm{e}}} \\
& +22989501.2(2.2) \mathrm{kHz}-310712.2294(17) \mathrm{kHz} .
\end{aligned}
$$

The first number in the second line of Eq. (30) is due to the difference of $E_{n \ell j}^{(\mathrm{fs})}$ for the two states, and includes the correlated error for the difference. The second term is due to the difference of $E_{n \ell j f}^{(\mathrm{hfs})}$ for the two states, and for these $1 \mathrm{~S}$ and $2 \mathrm{~S}$ states, $E^{(\mathrm{hfs})}$ is known precisely from the experimental results of Eq. (11). Solving for the Rydberg constant gives:

$$
\mathcal{R}=c R_{\infty}=3289841960248.9(3.0) \mathrm{kHz},
$$

where the uncertainty is dominated by the uncertainty in the $E^{(\mathrm{fs})}$ term. Eq. (31) differs considerably from the CODATA 2014 value of $3289841960355(19) \mathrm{kHz}$. The difference is almost entirely due to the fact that the CREMA value of $R_{\mathrm{p}}$ (Eq. (6) ) is used, whereas the CODATA compilation uses the value of Eq. (5). A very small contribution to the difference comes from the recent improvement [13] in the determination of $E^{(\mathrm{fs})}$ from the determination of $D_{71}^{(n \ell j)}$. The uncertainty in the CREMA value of $R_{\mathrm{p}}$ (Eq. (6) ) contributes $1.2 \mathrm{kHz}$ to the $3.0 \mathrm{kHz}$ uncertainty in Eq. (31), with the rest of the uncertainty resulting from the other (theoretical) uncertainties discussed in the last paragraph of Section III]

\section{TOTAL BINDING ENERGIES}

Using Eq. (91), along with the Rydberg constant of Eq. (31), the values of $E_{n \ell j}^{(\mathrm{fs})}$ of Section III and $E_{n \ell j f}^{(\mathrm{hfs})}$ of Section IV allows for a determination of the energies of all bound states of atomic hydrogen. Energies for $\ell=0$, 1 , and 2 are given in Tables III] IV] and V] respectively, with higher- $\ell$ energies given in Table VI. The uncertainties listed are dominated by the uncertainties in $E^{(\mathrm{fs})}$ (both due to the fine structure of the state and due to the effect of $E^{(\mathrm{fs})}$ on the determination of the Rydberg constant). The uncertainties listed take into account the correlation between these two ways that $E^{(\mathrm{fs})}$ enters the determination of the energies.

The final column in the tables gives the shift that the energy levels would experience if the CODATA 2014 value of $R_{\mathrm{p}}$ were used instead of the CREMA value. In referring to Eq. (19), one might assume that the value of $R_{\mathrm{p}}$ would affect only $\ell=0$ states. However, this is not the case, since the value of $R_{\mathrm{p}}$ also affects the determination 
TABLE III. Total binding energies for the lowest-lying $\mathrm{S}$ $(\ell=0)$ states, with uncertainties in the last digits given in parentheses. The last column gives the change $\delta_{R}$ in the binding energy that would result if $R_{\mathrm{p}}$ is increased by $0.03423 \mathrm{fm}$ (the difference between the CODATA and CREMA values).

\begin{tabular}{rrrc}
\hline \hline$n$ & $E\left(n \mathrm{~S}_{1 / 2} f=0\right)(\mathrm{kHz})$ & $E\left(n \mathrm{~S}_{1 / 2} f=1\right)(\mathrm{kHz})$ & $\delta_{R}(\mathrm{kHz})$ \\
\hline 1 & $-3288087922416.0(4)$ & $-3288086502010.2(4)$ & -15.3 \\
2 & $-822025577092.2(4)$ & $-822025399535.4(4)$ & -15.3 \\
3 & $-365343617904.3(2)$ & $-365343565294.9(2)$ & -8.5 \\
4 & $-205505309952.5(1)$ & $-205505287757.9(1)$ & -5.3 \\
5 & $-131523180988.2(1)$ & $-131523169624.6(1)$ & -3.6 \\
6 & $-91335431601.7(1)$ & $-91335425025.6(1)$ & -2.6 \\
7 & $-67103520641.6(1)$ & $-67103516500.4(1)$ & -1.9 \\
8 & $-51376096003.6(0)$ & $-51376093229.3(0)$ & -1.5 \\
9 & $-40593435126.6(0)$ & $-40593433178.1(0)$ & -1.2 \\
10 & $-32880666896.5(0)$ & $-32880665476.0(0)$ & -1.0 \\
11 & $-27174094155.3(0)$ & $-27174093088.1(0)$ & -0.8 \\
12 & $-22833779830.0(0)$ & $-22833779008.0(0)$ & -0.7 \\
13 & $-19455996185.2(0)$ & $-19455995538.7(0)$ & -0.6 \\
14 & $-16775829289.9(0)$ & $-16775828772.2(0)$ & -0.5 \\
15 & $-14613608126.8(0)$ & $-14613607705.9(0)$ & -0.4 \\
16 & $-12843989064.0(0)$ & $-12843988717.2(0)$ & -0.4 \\
17 & $-11377372464.9(0)$ & $-11377372175.8(0)$ & -0.4 \\
18 & $-10148333775.8(0)$ & $-10148333532.3(0)$ & -0.3 \\
19 & $-9108198599.8(0)$ & $-9108198392.7(0)$ & -0.3 \\
20 & $-8220148221.6(0)$ & $-8220148044.1(0)$ & -0.3 \\
\hline \hline
\end{tabular}

of the Rydberg constant (see Eq. (30)), and therefore, the energies of all states are affected.

\section{COMPARISON TO MEASURED VALUES}

Table VII gives a compilation of the most precise measurements to date of atomic hydrogen intervals. Many of these measurements reported values that were corrected for hyperfine structure. Given the inconsistency of hyperfine corrections applied in the literature (including inconsistent or incorrect application of anomalous moment corrections, of finite mass corrections and of corrections due to off-diagonal hyperfine-structure contributions), we have, where possible, given the actual intervals (or linear combination of intervals) measured.

Note that the hyperfine structure for the $4 \mathrm{P}_{1 / 2}, 4 \mathrm{P}_{3 / 2}$, $4 \mathrm{D}_{5 / 2}, 6 \mathrm{D}_{5 / 2}, 8 \mathrm{D}_{3 / 2}, 8 \mathrm{D}_{5 / 2}, 12 \mathrm{D}_{3 / 2}$, and $12 \mathrm{D}_{5 / 2}$ states (with splittings of $7396.2,2956.5,1140.5,337.9,221.8$, $142.6,65.7,42.2 \mathrm{kHz}$, respectively) is not resolved in the measurements of Table VII and therefore it is crucial to know both the hyperfine splittings and the relative strength of transitions to determine the energy splittings. Refs. [18, 22] give explicit values for the expected strength of the two hyperfine transitions $\left(\frac{2}{9}\right.$ and $\left.\frac{7}{9}\right)$ and give an explicit correction for how much this linear combination differs from the hyperfine centroid of the D states. Refs. 24, 25, 28] do not give such explicit corrections since their fits include the hyperfine structure. We therefore list the hyperfine centroid values given in those works. A further analysis of the work of Ref. 28] may be necessary, as they appear to use a simplified form for the presumed hyperfine intervals for their fits [32].

The third column of Table VII gives the prediction of this work for each of the measured intervals (or linear combination of intervals). Ten of the 15 entries in the table show agreement to within 1.5 standard deviations with the values given in this work. Four more of the entries agree to within 2 standard deviations. One measurement, (the $2 \mathrm{~S}$ to $12 \mathrm{D}_{5 / 2}$ interval, which is the most precise measurement in the table) disagrees by more than 3 standard deviations. The overall level of agreement between the measured values and the values of this work can be assessed by calculating a $\chi^{2}$ value for the agreement for the 15 entries in the table. The resultant $\chi^{2}$ of 30.6 shows that the agreement is not good. The $\chi^{2}$ distribution for 15 degrees of freedom has a probability of $0.10 \%$ for $\chi^{2}$ being 30.6 or larger (which is the equivalent of a 2.3-standard-deviation effect). This 2.3-standarddeviation discrepancy is dominated by the $2 \mathrm{~S}$ to $12 \mathrm{D}_{5 / 2}$ interval. If it were not included, the $\chi^{2}$ value would be 20.7 , which would be a 1.2-standard-deviation discrepancy.

The 2.3-standard-deviation discrepancy between column 2 and column 3 of Table VII could be eliminated if either the measurement of theoretical uncertainties are underestimated. In order to make the agreement acceptable, the theoretical uncertainty of column 3 would have to be increased by a factor of 20 . This could be achieved by assuming an uncertainty (cf. the last paragraph of Section III) of $(50 \mathrm{kHz}) \delta_{\ell 0} / n^{3}$. An overlooked contribution of this size seems unlikely given the many decades of careful work on atomic hydrogen theory. In order to get acceptable agreement, the measurement uncertainties would have to be increased by a factor of 2 . An increase in experimental uncertainties by a factor of 2 is far more plausible than an increase in theoretical uncertainties by a factor of 20 , especially given the large contribution of systematic effects in the measurements and given the fact that the measurement uncertainty is, in all cases, a very small fraction of the natural width of the transition. Ref. 33] discusses the tension between the most precise measurements in Table VII (and includes similar measurements in deuterium), and suggests that the tension might indicate an underestimation of experimental uncertainties.

Another way to analyze the discrepancy between column 2 and column 3 of Table VII is to determine the required change $\Delta R_{\mathrm{p}}$ in the proton radius (from its CREMA value) that would lead to agreement between the values in these columns. These $\Delta R_{\mathrm{p}}$ values are given in the final column of Table VII along with their uncertainties (which are dominated by the measurement uncertainties of column 2). The listed values of $\Delta R_{\mathrm{p}}$ are almost all positive, and their weighted average is $0.035(7) \mathrm{fm}$ (with an acceptable $\chi^{2}$ value of 7.3 for 14 degrees of freedom). Thus, the atomic hydrogen data would be consistent with the theoretical predictions if $R_{\mathrm{p}}$ were $4 \%$ larger than the CREMA value. 
TABLE IV. Continuation of Table $\amalg$ for the lowest-lying $\mathrm{P}(\ell=1)$ states,

\begin{tabular}{rlllll}
\hline \hline$n$ & $E\left(n \mathrm{P}_{1 / 2} f=0\right)(\mathrm{kHz})$ & $E\left(n \mathrm{P}_{1 / 2} f=1\right)(\mathrm{kHz})$ & $E\left(n \mathrm{P}_{3 / 2} f=1\right)(\mathrm{kHz})$ & $E\left(n \mathrm{P}_{3 / 2} f=2\right)(\mathrm{kHz})$ & $\delta_{R}(\mathrm{kHz})$ \\
\hline 2 & $-822026546135.9(7)$ & $-822026486966.4(7)$ & $-822015547496.7(7)$ & $-822015523845.1(7)$ \\
3 & $-365343906471.0(3)$ & $-365343888939.2(3)$ & $-365340647611.9(3)$ & $-365340640604.0(3)$ & -26.8 \\
4 & $-205505431929.9(2)$ & $-205505424533.7(2)$ & $-205504057100.4(2)$ & $-205504054143.9(2)$ & -6.7 \\
5 & $-131523243500.5(1)$ & $-131523239713.6(1)$ & $-131522539588.6(1)$ & $-131522538074.9(1)$ & -4.3 \\
6 & $-91335467797.2(1)$ & $-91335465605.8(1)$ & $-91335060441.3(1)$ & $-91335059565.3(1)$ & -3.0 \\
7 & $-67103543442.8(1)$ & $-67103542062.8(1)$ & $-67103286915.7(1)$ & $-67103286364.0(1)$ & -2.2 \\
8 & $-51376111281.9(0)$ & $-51376110357.4(0)$ & $-51375939428.9(0)$ & $-51375939059.3(0)$ & -1.7 \\
9 & $-40593445858.7(0)$ & $-40593445209.3(0)$ & $-40593325160.9(0)$ & $-40593324901.3(0)$ & -1.3 \\
10 & $-32880674721.0(0)$ & $-32880674247.6(0)$ & $-32880586732.3(0)$ & $-32880586543.1(0)$ & -1.1 \\
11 & $-27174100034.5(0)$ & $-27174099678.8(0)$ & $-27174033927.3(0)$ & $-27174033785.2(0)$ & -0.9 \\
12 & $-22833784358.7(0)$ & $-22833784084.8(0)$ & $-22833733439.4(0)$ & $-22833733329.9(0)$ & -0.7 \\
13 & $-19455999747.3(0)$ & $-19455999531.9(0)$ & $-19455959697.9(0)$ & $-19455959611.8(0)$ & -0.6 \\
14 & $-16775832142.0(0)$ & $-16775831969.5(0)$ & $-16775800076.2(0)$ & $-16775800007.3(0)$ & -0.5 \\
15 & $-14613610445.7(0)$ & $-14613610305.5(0)$ & $-14613584375.1(0)$ & $-14613584319.0(0)$ \\
\hline \hline
\end{tabular}

TABLE V. Continuation of Tables III and IV for the lowest-lying D $(\ell=2)$ states.

\begin{tabular}{rlllll}
\hline \hline$n$ & $E\left(n \mathrm{D}_{3 / 2} f=1\right)(\mathrm{kHz})$ & $E\left(n \mathrm{D}_{3 / 2} f=2\right)(\mathrm{kHz})$ & $E\left(n \mathrm{D}_{5 / 2} f=2\right)(\mathrm{kHz})$ & $E\left(n \mathrm{D}_{5 / 2} f=3\right)(\mathrm{kHz})$ & $\delta_{R}(\mathrm{kHz})$ \\
\hline 3 & $-365340651193.1(3)$ & $-365340646986.5(3)$ & $-365339566803.7(3)$ & $-365339564100.3(3)$ \\
4 & $-205504058649.5(2)$ & $-205504056874.8(2)$ & $-205503601172.2(2)$ & $-205503600031.7(2)$ & -11.9 \\
5 & $-131522540392.4(1)$ & $-131522539483.7(1)$ & $-131522306163.9(1)$ & $-131522305580.0(1)$ \\
6 & $-91335060910.1(1)$ & $-91335060384.3(1)$ & $-91334925361.2(1)$ & $-91334925023.3(1)$ & -4.3 \\
7 & $-67103287212.4(1)$ & $-67103286881.3(1)$ & $-67103201852.2(1)$ & $-67103201639.4(1)$ & -3.0 \\
8 & $-51375939628.3(0)$ & $-51375939406.5(0)$ & $-51375882443.7(0)$ & $-51375882301.1(0)$ & -1.7 \\
9 & $-40593325301.3(0)$ & $-40593325145.5(0)$ & $-40593285138.7(0)$ & $-40593285038.6(0)$ & -1.3 \\
10 & $-32880586834.9(0)$ & $-32880586721.3(0)$ & $-32880557556.4(0)$ & $-32880557483.4(0)$ & -1.1 \\
11 & $-27174034004.5(0)$ & $-27174033919.1(0)$ & $-27174012007.1(0)$ & $-27174011952.2(0)$ & -0.9 \\
12 & $-22833733498.9(0)$ & $-22833733433.2(0)$ & $-22833716555.3(0)$ & $-22833716513.1(0)$ \\
\hline \hline
\end{tabular}

TABLE VI. Continuation of Tables III IV and $\mathrm{V}$ for the lowest-lying $(\ell \geq 3)$ states.

\begin{tabular}{|c|c|c|c|c|c|c|}
\hline$n$ & $\ell$ & $E\left(n \ell j=\ell-\frac{1}{2} f=\ell-1\right)$ & $E\left(n \ell j=\ell-\frac{1}{2} f=\ell\right)$ & $E\left(n \ell j=\ell+\frac{1}{2} f=\ell\right)$ & $E\left(n \ell j=\ell+\frac{1}{2} f=\ell+1\right)$ & $\delta_{R}(\mathrm{kHz})$ \\
\hline 4 & 3 & $-205503601791.5(2)$ & $-205503600976.7(2)$ & $-205503373140.3(2)$ & $-205503372536.9(2)$ & -6.7 \\
\hline 5 & 3 & $-131522306484.4(1)$ & $-131522306067.2(1)$ & $-131522189415.0(1)$ & $-131522189106.0(1)$ & -4.3 \\
\hline 6 & 3 & -91334925 548.0(1) & $-91334925306.5(1)$ & -91334857 799.4(1) & -91334857620.6(1) & -3.0 \\
\hline 8 & 3 & $-51375882523.1(0)$ & $-51375882421.2(0)$ & $-51375853941.6(0)$ & $-51375853866.2(0)$ & -1.7 \\
\hline 9 & 3 & $-40593285194.6(0)$ & $-40593285123.0(0)$ & $-40593265120.9(0)$ & $-40593265067.9(0)$ & -1.3 \\
\hline 5 & 4 & $-131522189594.9(1)$ & $-131522189354.5(1)$ & $-131522119365.0(1)$ & $-131522119173.9(1)$ & -4.3 \\
\hline 8 & 4 & $-51375853986.1(0)$ & $-51375853927.4(0)$ & $-51375836840.1(0)$ & $-51375836793.4(0)$ & -1.7 \\
\hline 9 & 4 & $-40593265152.2(0)$ & -40593265 111.0(0) & $-40593253110.0(0)$ & -40593253077.2(0) & -1.3 \\
\hline 6 & 5 & $-91334817329.4(1)$ & -91334817 238.9(1) & -91334790237.1(1) & -91334790161.9(1) & -3.0 \\
\hline 7 & 5 & $-67103133821.4(1)$ & $-67103133764.4(1)$ & $-67103116760.4(1)$ & $-67103116713.0(1)$ & -2.2 \\
\hline 8 & 5 & $-51375836868.8(0)$ & $-51375836830.6(0)$ & $-51375825439.2(0)$ & $-51375825407.5(0)$ & -1.7 \\
\hline 8 & 7 & $-51375817310.8(0)$ & $-51375817290.9(0)$ & $-51375811188.4(0)$ & $-51375811171.0(0)$ & -1.7 \\
\hline 9 & 7 & $-40593239394.0(0)$ & $-40593239380.0(0)$ & $-40593235094.1(0)$ & $-40593235081.9(0)$ & -1.3 \\
\hline 9 & 8 & $-40593235102.2(0)$ & $-40593235091.4(0)$ & $-40593231757.9(0)$ & $-40593231748.3(0)$ & -1.3 \\
\hline
\end{tabular}

This $4 \%$ discrepancy has been the topic of great interest since the muonic measurement of $R_{\mathrm{p}}$ was published
[3]. A similar discrepancy has been found between the CREMA value for $R_{\mathrm{p}}$ and that obtained from electron 
TABLE VII. Comparison to Measurements. Column 2 gives the measured interval (or linear combination of intervals), and column 3 gives the predicted interval from this work. The final column gives the amount by which the proton radius would have to change in order to give agreement between column 2 and 3. One standard deviation uncertainties are given in parentheses.

\begin{tabular}{|c|c|c|c|}
\hline (or combinations of intervals) & Measurement $(\mathrm{kHz})$ & This Work $(\mathrm{kHz})$ & $\Delta R_{\mathrm{p}}(\mathrm{fm})$ \\
\hline$\left(2 \mathrm{~S}_{\frac{1}{2}} f=1 \rightarrow 4 \mathrm{~S}_{\frac{1}{2}} f=1\right)-\frac{1}{4}\left(1 \mathrm{~S}_{\frac{1}{2}} f=1 \rightarrow 2 \mathrm{~S}_{\frac{1}{2}} f=1\right)$ & $4836176(10)^{\mathrm{a}}$ & $4836158.8(3)$ & $+0.059(34)$ \\
\hline$\frac{2}{9}\left(2 \mathrm{~S}_{\frac{1}{2}} f=1 \rightarrow 4 \mathrm{D}_{\frac{5}{2}} f=2\right)+\frac{7}{9}\left(2 \mathrm{~S}_{\frac{1}{2}} f=1 \rightarrow 4 \mathrm{D}_{\frac{5}{2}} f=3\right)-\frac{1}{4}\left(1 \mathrm{~S}_{\frac{1}{2}} f=1 \rightarrow 2 \mathrm{~S}_{\frac{1}{2}} f=1\right)$ & $55(24)^{\mathrm{b}}$ & $6523631.6(2)$ & $+0.093(95)$ \\
\hline $2 \mathrm{~S}_{\frac{1}{2}} f^{2}=1 \rightarrow 8 \mathrm{~S}_{\frac{1}{2}} f^{2}=1 \quad{ }^{2}$ & $770649306316.4(8.6)^{\mathrm{c}}$ & $770649306306.1(4)$ & $+0.025(21)$ \\
\hline$\frac{3}{8}\left(2 \mathrm{~S}_{\frac{1}{2}} f=1 \rightarrow 8 \mathrm{D}_{\frac{3}{2}} f=1\right)+\frac{5}{8}\left(2 \mathrm{~S}_{\frac{1}{2}} f=1 \rightarrow 8 \mathrm{D}_{\frac{3}{2}} f=2\right)$ & $770649460060.8(8.3)^{\mathrm{d}}$ & $770649460045.7(4)$ & $+0.038(21)$ \\
\hline$\frac{5}{12}\left(2 \mathrm{~S}_{\frac{1}{2}}^{2} f=1 \rightarrow 8 \mathrm{D}_{\frac{5}{2}}^{2} f=2\right)+\frac{7}{12}\left(2 \mathrm{~S}_{\frac{1}{2}}^{2} f=1 \rightarrow 8 \mathrm{D}_{\frac{5}{2}}^{2} f=3\right)$ & $770649517195.0(6.4)^{\mathrm{e}}$ & $770649517174.9(4)$ & $+0.051(16)$ \\
\hline$\frac{3}{8}\left(2 \mathrm{~S}_{\frac{1}{2}}{ }^{2} f=1 \rightarrow 12 \mathrm{D}_{\frac{3}{2}}^{2} f=1\right)+\frac{5}{8}\left(2 \mathrm{~S}_{\frac{1}{2}}{ }^{2} f=1 \rightarrow 12 \mathrm{D}_{\frac{3}{2}}^{2} f=2\right)$ & $799191666083.5(9.3)^{\mathrm{f}}$ & $799191666077.6(4)$ & +0.0 \\
\hline$\frac{5}{12}\left(2 \mathrm{~S}_{\frac{1}{2}}^{2} f=1 \rightarrow 12 \mathrm{D}_{\frac{5}{2}}^{2} f=2\right)+\frac{7}{12}\left(2 \mathrm{~S}_{\frac{1}{2}}^{2} f=1 \rightarrow 12 \mathrm{D}_{\frac{5}{2}}^{2} f=3\right)$ & $799191683014.5(7.0)^{\mathrm{g}}$ & $799191683004.7(4)$ & $+0.023(16)$ \\
\hline $1 \mathrm{~S}_{\frac{1}{2}} f=1 \rightarrow 3 \mathrm{~S}_{\frac{1}{2}} f=1^{2}$ & $2922742936729(13)^{\mathrm{h}}$ & $2922742936715.0(2)$ & $+0.069(65)$ \\
\hline$\left(2 \mathrm{~S}_{\frac{1}{2}}^{2} f=1 \rightarrow 6 \mathrm{~S}_{\frac{1}{2}}^{2} f=1\right)-\frac{1}{4}\left(1 \mathrm{~S}_{\frac{1}{2}} f=1 \rightarrow 3 \mathrm{~S}_{\frac{1}{2}} f=1\right)$ & $4240346(21)^{\mathrm{i}}$ & $4240331.0(3)$ & $+0.047(65)$ \\
\hline$\frac{5}{12}\left(2 \mathrm{~S}_{\frac{1}{2}} f=1 \rightarrow 6 \mathrm{D}_{\frac{5}{2}}^{2} f=2\right)+\frac{7}{12}\left(2 \mathrm{~S}_{\frac{1}{2}} f=1 \rightarrow{ }^{2} \mathrm{D}_{\frac{5}{2}} f=3\right)-\frac{1}{4}\left(1 \mathrm{~S}_{\frac{1}{2}} f=1 \rightarrow 3 \mathrm{~S}_{\frac{1}{2}} f=1\right.$ & $4740197(11)^{\bar{j}}$ & $4740192.5(3)$ & +0.0 \\
\hline$\frac{1}{4}\left(2 \mathrm{~S}_{\frac{1}{2}}^{2} f=1 \rightarrow 4 \mathrm{P}_{\frac{1}{2}}^{2} f=0\right)+\frac{3}{4}\left(2 \mathrm{~S}_{\frac{1}{2}} f^{2}=1 \rightarrow 4 \mathrm{P}_{\frac{1}{2}} f^{2}=1\right)-\frac{1}{4}\left(1 \mathrm{~S}_{\frac{1}{2}} f^{2}=1 \rightarrow 2 \mathrm{~S}_{\frac{1}{2}} f^{2}=1\right)$ & $4697560.0(14.9)^{\mathrm{k}}$ & $4697534.0(2)$ & $+0.104(59)$ \\
\hline$\frac{3}{8}\left(2 \mathrm{~S}_{\frac{1}{2}}^{2} f=1 \rightarrow 4 \mathrm{P}_{\frac{3}{2}}^{2} f=1\right)+\frac{5}{8}\left(2 \mathrm{~S}_{\frac{1}{2}}^{2} f=1 \rightarrow 4 \mathrm{P}_{\frac{3}{2}}^{2} f=2\right)-\frac{1}{4}\left(1 \mathrm{~S}_{\frac{1}{2}}^{2} f=1 \rightarrow 2 \mathrm{~S}_{\frac{1}{2}}^{2} f=1\right)$ & $6068664.0(10.5)^{1}$ & $6068664.1(2)$ & -0.0 \\
\hline $2 \mathrm{~S}_{\frac{1}{2}} f^{2}=0 \rightarrow 2 \mathrm{P}_{\frac{3}{2}} f^{2}=1$ & $10029586(12)^{\mathrm{m}}$ & $10029595.6(3)$ & $+0.029(36)$ \\
\hline $2 \mathrm{P}_{\frac{1}{2}}^{2} f=1 \rightarrow 2 \mathrm{~S}_{\frac{1}{2}}^{2} f=0$ & $909887(9)^{\mathrm{n}}$ & $909874.2(3)$ & $+0.038(27)$ \\
\hline $2 \mathrm{P}_{\frac{1}{2}}^{2} f=1 \rightarrow 2 \mathrm{~S}_{\frac{1}{2}}^{2} f=0$ & $909904(20)^{\circ}$ & $909874.2(3)$ & $+0.089(60)$ \\
\hline
\end{tabular}

${ }^{a}$ Ref. [18] with their hfs correction of $-38838 \mathrm{kHz}$ subtracted out to give the original measured value.

b Refs. 18, 22 with their hfs correction of $-33.511 \mathrm{kHz}$ subtracted out. The $4 \mathrm{D}_{5 / 2} \mathrm{hfs}$ is not resolved in the measurement.

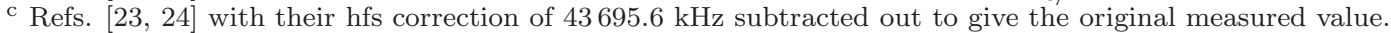

d Refs. 23, 24 with their hfs correction of $44389.2 \mathrm{kHz}$ subtracted out. The $8 \mathrm{D}_{3 / 2} \mathrm{hfs}$ is not resolved in the measurement.

e Refs. 23, 24] with their hfs correction of $44389.2 \mathrm{kHz}$ subtracted out. The $8 \mathrm{D}_{5 / 2} \mathrm{hfs}$ is not resolved in the measurement.

${ }^{f}$ Refs. 24, 25] with their hfs correction of $44389.2 \mathrm{kHz}$ subtracted out. The $12 \mathrm{D}_{3 / 2} \mathrm{hfs}$ is not resolved in the measurement.

g Refs. 24, 25] with their hfs correction of $44389.2 \mathrm{kHz}$ subtracted out. The $12 \mathrm{D}_{5 / 2} \mathrm{hfs}$ is not resolved in the measurement.

h Ref. 26.

i Ref. 24, 27 with their hfs correction of $-42742.1 \mathrm{kHz}$ subtracted out to give the original measured value.

${ }^{j}$ Ref. 24, 27] with their hfs correction of $-41098.1 \mathrm{kHz}$ subtracted out. The $8 \mathrm{D}_{5 / 2} \mathrm{hfs}$ is not resolved in the measurement.

${ }^{\mathrm{k}}$ Ref. 28] with their hfs correction of $-33291 \mathrm{kHz}$ subtracted out. The $4 \mathrm{P}_{1 / 2} \mathrm{hfs}$ is not resolved in the measurement.

${ }^{1}$ Ref. 28] with their hfs correction of $-33291 \mathrm{kHz}$ subtracted out. The $4 \mathrm{P}_{3 / 2} \mathrm{hfs}$ is not resolved in the measurement.

$\mathrm{m}$ Ref. 29] with their hfs correction of $-118386 \mathrm{kHz}$ subtracted out to give the original measured value.

${ }^{n}$ Ref. 301.

${ }^{\circ}$ Ref. 31].

proton scattering [34 36] (although our recent analysis 37] of the scattering data does not support the notion of this discrepancy). The $4 \%$ discrepancy for $R_{\mathrm{p}}$ is often referred to as the proton size puzzle, and many works have discussed the puzzle (see Refs. 38 [40] for reviews of this body of work).

\section{SUMMARY}

We present clear formulas and tabulations of the bound-state energy levels for atomic hydrogen. The tabulation includes the new, more precise value for the proton charge radius. The hyperfine structure corrections due to the anomalous moment and the finite mass of the proton, and due to off-diagonal matrix elements of the hyperfine Hamiltonian are clearly laid out, along with general formulas for their dependence on $n, \ell, j$, and $f$. The energy of all bound states of atomic hydrogen can now be determined to a precision of better than $1 \mathrm{kHz}$.

This work is supported by NSERC and CRC.
[1] P. J. Mohr, B. N. Taylor, and D. B. Newell, Journal of Physical and Chemical Reference Data, 41, 043109 (2012).

[2] A. Antognini, F. Nez, K. Schuhmann, F. D. Amaro, F. Biraben, J. M. Cardoso, D. S. Covita, A. Dax,
S. Dhawan, M. Diepold, et al., Science, 339, 417 (2013). [3] R. Pohl, A. Antognini, F. Nez, F. D. Amaro, F. Biraben, J. M. Cardoso, D. S. Covita, A. Dax, S. Dhawan, L. M. Fernandes, et al., Nature, 466, 213 (2010).

[4] C. G. Parthey, A. Matveev, J. Alnis, B. Bernhardt, 
A. Beyer, R. Holzwarth, A. Maistrou, R. Pohl, K. Predehl, T. Udem, et al., Physical Review Letters, 107, 203001 (2011).

[5] N. Kolachevsky, A. Matveev, J. Alnis, C. G. Parthey, S. G. Karshenboim, and T. W. Hänsch, Physical Review Letters, 102, 213002 (2009).

[6] S. G. Karshenboim, Physics Reports, 422, 1 (2005).

[7] G. W. Drake and R. A. Swainson, Physical Review A, 41, 1243 (1990).

[8] U. D. Jentschura, Physical Review A, 74, 062517 (2006).

[9] U. D. Jentschura and P. J. Mohr, Physical Review A, 72, 014103 (2005).

[10] U. D. Jentschura and P. J. Mohr, Physical Review A, 69, 064103 (2004).

[11] U. D. Jentschura, Journal of Physics A, 36, L229 (2003).

[12] S. Kotochigova, P. J. Mohr, and B. N. Taylor, Canadian Journal of Physics, 80, 1373 (2002).

[13] V. Yerokhin and V. Shabaev, Physical Review Letters, 115, 233002 (2015).

[14] U. D. Jentschura and V. A. Yerokhin, Physical Review A, 73, 062503 (2006).

[15] S. J. Brodsky and R. G. Parsons, Physical Review, 163, 134 (1967).

[16] B. Wundt and U. Jentschura, Physical Review A, 83, 052501 (2011).

[17] A. Kramida, Atomic Data and Nuclear Data Tables, 96, $586(2010)$.

[18] M. Weitz, A. Huber, F. Schmidt-Kaler, D. Leibfried, W. Vassen, C. Zimmermann, K. Pachucki, T. Hänsch, L. Julien, and F. Biraben, Physical Review A, 52, 2664 (1995).

[19] A. Martynenko, Physics of Atomic Nuclei, 71, 125 (2008).

[20] U. D. Jentschura, Annals of Physics, 326, 500 (2011).

[21] B. Wundt and U. Jentschura, Journal of Physics B, 43, $115002(2010)$.

[22] M. Weitz, F. Schmidt-Kaler, and T. Hänsch, Solid State Lasers: New Developments and Applications, 317, 331 (1993).

[23] B. De Beauvoir, F. Nez, L. Julien, B. Cagnac, F. Biraben, D. Touahri, L. Hilico, O. Acef, A. Clairon, and J. Zondy, Physical Review Letters, 78, 440 (1997).

[24] B. De Beauvoir, C. Schwob, O. Acef, L. Jozefowski, L. Hilico, F. Nez, L. Julien, A. Clairon, and F. Biraben, European Physical Journal D, 12, 61 (2000).

[25] C. Schwob, L. Jozefowski, B. De Beauvoir, L. Hilico,
F. Nez, L. Julien, F. Biraben, O. Acef, J.-J. Zondy, and A. Clairon, Physical Review Letters, 82, 4960 (1999).

[26] O. Arnoult, F. Nez, L. Julien, and F. Biraben, European Physical Journal D, 60, 243 (2010).

[27] S. Bourzeix, B. De Beauvoir, F. Nez, M. Plimmer, F. De Tomasi, L. Julien, F. Biraben, and D. Stacey, Physical Review Letters, 76, 384 (1996).

[28] D. Berkeland, E. Hinds, and M. Boshier, Physical Review Letters, 75, 2470 (1995).

[29] E. Hagley and F. Pipkin, Physical Review Letters, 72, 1172 (1994).

[30] S. Lundeen and F. Pipkin, Physical Review Letters, 46, 232 (1981).

[31] G. Newton, D. Andrews, and P. Unsworth, Philosophical Transactions of the Royal Society of London A: Mathematical, Physical and Engineering Sciences, 290, 373 (1979).

[32] D. J. Berkeland, Dissertation, Yale University, 1995.

[33] S. G. Karshenboim, Physical Review A, 91, 012515 (2015).

[34] J. C. Bernauer, P. Achenbach, C. Ayerbe Gayoso, R. Böhm, D. Bosnar, L. Debenjak, M. O. Distler, L. Doria, A. Esser, H. Fonvieille, J. M. Friedrich, J. Friedrich, M. Gómez Rodríguez de la Paz, M. Makek, H. Merkel, D. G. Middleton, U. Müller, L. Nungesser, J. Pochodzalla, M. Potokar, S. Sánchez Majos, B. S. Schlimme, S. Sirca, T. Walcher, and M. Weinriefer, Physical Review Letters, 105, 242001 (2010).

[35] J. Bernauer, M. Distler, J. Friedrich, T. Walcher, P. Achenbach, C. A. Gayoso, R. Böhm, D. Bosnar, L. Debenjak, L. Doria, et al., Physical Review C, 90, 015206 (2014).

[36] G. Lee, J. R. Arrington, and R. J. Hill, Physical Review D, 92, 013013 (2015).

[37] M. Horbatsch and E. Hessels, arXiv preprint arXiv:1509.05644 (2015).

[38] R. Pohl, R. Gilman, G. A. Miller, and K. Pachucki, Annual Review of Nuclear and Particle Science, 63, 175 (2013).

[39] J. C. Bernauer and R. Pohl, Scientific American, 310, 32 (2014).

[40] C. E. Carlson, Progress in Particle and Nuclear Physics, 82, 59 (2015). 